\title{
Appraising guidelines: the AGREE Instrument
}

\section{Derek Richards

\author{
Director, Centre for Evidence-based Dentistry, Oxford, UK
}

\begin{abstract}
As clinicians we all have routines for the management of the common clinical problems we encounter day in day out. These routines are developed by clinicians reproducing the advice and instructions of our teachers and colleagues. This process is or should be modified and updated by advances in treatment regimes and diagnostic techniques. For less common conditions we might look to a textbook or some guidelines as to how to proceed. However with increasing numbers of guidelines now available how do we tell which ones are valid, or whether you should follow a particular guideline?
\end{abstract}

Evidence-Based Dentistry (2002) 3, 109-110. doi:10.1038/sj.ebd.6400140

The number and availability of guidelines is increasing and they cover not only rarer conditions ${ }^{1}$ but also treatment for common conditions, eg, Preventing Dental Caries in Children at High Caries Risk. ${ }^{2}$ An impression of how many guidelines are available in dentistry can be gleaned from visiting the guidelines database of the FDI World Dental Federation (www.fdiworldental.org/resources/index.htm).

Clinical practice guidelines are "systematically developed statements to assist practitioner and patient decisions about appropriate health care for specific clinical circumstances". ${ }^{3}$ The aim is to make explicit recommendations with the aim of influencing clinicians' behaviour. Clinical practice guidelines are also being used to improve the quality of patient care, eg, Selection Criteria for Dental Radiography. ${ }^{4}$ There is concern, however, about the number of low-quality guidelines, conflicting recommendations and duplication of effort. ${ }^{5,6,7,8}$ A common, valid and transparent approach for the development of good clinical practice guide- lines is required. One group working within the UK that has such an approach is the Scottish Intercollegiate Guideline Network (SIGN) which has produced two dental guidelines. ${ }^{2,9}$

SIGN are members of collaboration of researchers and policymakers who have been looking to improve the quality and effectiveness of clinical practice guidelines by establishing a shared framework for their development, reporting and assessment. This group is called AGREE: Appraisal of Guidelines for Research and Evaluation. One of the products of the AGREE collaboration is a generic tool, the AGREE Instrument. This instrument is designed primarily to help guideline developers and users assess the methodological quality of clinical practice guidelines.

By quality of clinical practice guidelines, AGREE mean "the confidence that the potential biases of guideline development have been addressed adequately and that the recommendations are both internally and externally valid, and are feasible for practice. This process involves taking into account the benefits, harms and costs of the recommendations, as well as the practical issues attached to them." Therefore, the AGREE instrument assesses the methods used for developing the guidelines, the content of the final recommendations, and the factors linked to their uptake. The full instrument can be downloaded from the AGREE website (www.agreecollaboration.org/). The instrument consists of 23 key items organised in six domains looking at difference dimensions of guideline quality. The domains are:

- Scope and Purpose (items 1-3) is concerned with the overall aim of the guideline, the specific clinical questions and the target patient population.

- Stakeholder Involvement (items 47) focuses on the extent to which the guideline represents the views of its intended users.

- Rigour of Development (items 814) relates to the process used to gather and synthesise the evidence, the methods to formulate the recommendations and to update them.

- Clarity and Presentation (items 1518) deals with the language and format of the guideline.

- Applicability (items 19-21) pertains to the likely organisational, behavioural and cost implications of applying the guideline.

- Editorial Independence (items 2223) is concerned with the independence of the recommendations and 
acknowledgement of possible conflict of interest from the guideline development group.

The AGREE instrument also provides more detailed information about the response scales for the items and user guides to each domain. A section for overall assessment is included at the end, which requires the appraiser to make a judgement as to the quality of the guideline, taking each of the appraisal criteria into account.

Much effort and resources are currently expended on the production of guidelines in dentistry. Canada has recently established the Canada Collaboration on Clinical Practice Guidelines in Dentistry (CCCD) (www.cccd.ca/) to develop clinical practice guidelines building on work previously been carried out there with medical guidelines (www.cma.ca/cma/common/start.do?lang=2). The CCCD's guidelines are being developed in accordance with the recommendations of the AGREE collaboration. Other guidelines sites can be found by visiting the Centre for Evidence-based Dentistry guidelines page (www.ihs.ox.ac.uk/cebd/guidelines.htm). Increasingly these guidelines are evidence-based - but there still remain problems with duplication of effort. This can be witnessed by observing the number of guidelines available for third molars or the use of fluorides (see FDI World Dental Federation guidelines website).

The quality of evidence supporting the practice of dentistry is patchy so the evidence base to support guidelines is not too deep to fathom. The production of good-quality guidelines therefore should be of benefit to practitioners who have limited time to assess all the available information. Those producing guidelines in dentistry, however, should look to using the quality framework established by AGREE and systematic reviews available from the Cochrane Collaboration, or conduct systematic reviews using the Cochrane Collaboration (www.cochrane.org/cochrane/hbook.htm) or the York Centre for Reviews and Dissemination methodology ${ }^{10}$ as the basis for their guidelines. Producing guidelines in this manner will help practitioners and patients make decisions about appropriate health care in specific clinical circumstances.

Guideline developers also need to consider that producing the guideline is not the end of the process. Sound guideline development is the beginning: implementation, dissemination and updating are also important. Sadly, this is often forgotten. An example of this is the Selection Criteria for Dental Radiography produced by the Faculty of General Dental Practitioners (UK). This was a good guideline produced using the SIGN methodology but poor dissemination and implementation means that there was little uptake. This guideline is now in need of an update and a current European project is looking at a wider project on medical radiography.

Guidelines can assist the practitioner and patient to achieve the most appropriate care but we are overwhelmed with them. The use of a critical appraisal tool such as the AGREE instrument is therefore an important aid in the selection of the best-quality guidelines for use in practice. Ideally practitioners should be involved in guideline development: this has been shown to improve uptake and use.
1. Zakrzewska JM. Cluster headache: a review of the literature $\mathrm{Br} J$ Oral Maxillofacial Surg 2001: 39:103-113.

2. Scottish Intercollegiate Guideline Network. Preventing Dental Caries in Children at High Caries Risk. Targeted Prevention of Dental Caries in the Permanent Teeth of 616-Year Olds Presenting for Dental Care.

Publication No. 47; 2000. ISBN 189989332

6 (www.sign.ac.uk/guidelines/fulltext/47/ index.html)

3. Field MJ, Lohr KN (Editors). Institute of Medicine Committee to Advise the Public Health Service on Clinical Practice Guidelines: Directions For a New Programme. Washington DC: National Academy Press; 1990.

4. Faculty of General Dental Practitioners (UK). Selection Criteria for Dental Radiography. London Royal College of Surgeons of England. ISBN 0952053799.

5. Shaneyfelt TM, Mayo-Smith MF, Rothwang I. Are guidelines following guidelines? The methodological quality of clinical practice guidelines in the peerreviewed medical literature. J Am Med Assoc 1999; 281:1900-1905.

6. Grilli R, Magrini N, Penna A, Mura G, Liberati A. Practice guidelines developed by specialty societies: the need for a critical appraisal. Lancet 2000; 355:103-105.

7. Fahey T, Peters TJ. Clinical guidelines and the management of hypertension: a between-practice and guideline comparison. Br J Gen Practice 1997; 47:729-730.

8. Thomson $R$, McElroy $H$, Sudlow $M$. Guidelines on anticoagulant treatment in atrial fibrillation in Great Britain: variation in content and implications for treatment. $\mathrm{Br}$ Med J 1998; 316:509-513.

9. Scottish Intercollegiate Guideline Network. Management of Unerupted and Impacted Third Molar Teeth Publication No. 43; 2000. ISBN 1899893121 (www.sign.ac.uk/guidelines/fulltext/43/ index.html).

10. NHS Centre for Reviews and Dissemination. Undertaking Systematic Reviews of Research on Effectiveness: CRD's Guidance for Carrying Out or Commissioning Reviews, 2nd Edn. York: University of York NHS Centre for Reviews and Dissemination; 2001. 